Pacific

Journal of

Mathematics

\title{
ANALYTIC PROPERTIES OF DIRICHLET SERIES OBTAINED FROM THE ERROR TERM IN THE DIRICHLET DIVISOR PROBLEM
}

JUN FURUYA AND YoshIO TANIGAWA 


\title{
ANALYTIC PROPERTIES OF DIRICHLET SERIES OBTAINED FROM THE ERROR TERM IN THE DIRICHLET DIVISOR PROBLEM
}

\author{
JUN FURUYA AND YOSHIO TANIGAWA
}

We discuss some analytic properties of Dirichlet series

$$
Y(s)=\sum_{n=1}^{\infty} d(n) \Delta(n) n^{-s} \quad \text { for } \operatorname{Re} s>\frac{5}{4},
$$

where $d(n)$ is the divisor function and $\Delta(x)$ is the error term in the Dirichlet divisor problem. In particular, we study an analytic continuation and an order of $Y(s)$. As applications, we study an analytic continuation and orders of several kinds of Dirichlet series related to $\Delta(x)$.

\section{Introduction and statement of results}

Let $d(n)$ be the divisor function, and let $\Delta(x)$ be the error term in the Dirichlet divisor problem, defined by

$$
\Delta(x)=\sum_{n \leq x} d(n)-x(\log x+2 \gamma-1),
$$

where $\gamma$ is the Euler constant. A long history of research on $\Delta(x)$ has not settled the famous conjecture that $\Delta(x)=O\left(x^{1 / 4+\varepsilon}\right)$, where $\varepsilon$ is an arbitrarily small positive number. An efficient way to investigate $\Delta(x)$ is to consider the Dirichlet series whose coefficients involve $\Delta(x)$ or the related integrals.

In [Furuya et al. 2010], we considered properties of the Dirichlet series $D_{j}(s)$ defined by

$$
D_{j}(s)=\sum_{n=1}^{\infty} \frac{\Delta(n)^{j}}{n^{s}},
$$

for $j=1$ and 2. It is easily seen that these functions are absolutely convergent for $\sigma>5 / 4$ for $j=1$ and $\sigma>3 / 2$ for $j=2$. Here, and in what follows, we denote the

MSC2000: 11L07, 11M41.

Keywords: analytic continuation, Dirichlet series, divisor problem, double zeta function, orders of

Dirichlet series.

Furuya is supported by Grant-in-Aid for Scientific Research number 18740022. 
complex number $s$ as $s=\sigma+i t$ with real numbers $\sigma$ and $t$. We have established the analytic continuation and the locations of poles of these functions:

Theorem [Furuya et al. 2010, Theorems 1 and 2]. The function $D_{1}(s)$ can be continued to the whole complex plane as a meromorphic function. This function has a double pole at $s=1$ and a simple pole at $s=-2 n$ with a nonnegative integer $n$. In particular, the Laurent expansion of $D_{1}(s)$ at $s=1$ is given by

$$
D_{1}(s)=\frac{1}{2(s-1)^{2}}+\frac{\gamma+\frac{1}{4}}{s-1}+O(1) .
$$

The function $D_{2}(s)$ can be continued to the region $\operatorname{Re} s>2 / 3$ as a meromorphic function. This function has a simple pole at $s=3 / 2$ and a triple pole at $s=1$.

One of the results in [Furuya et al. 2010] is the relationship between the Dirichlet series $D_{2}(s)$ and Lau and Tsang's conjecture [1995, Formula 1.3],

$$
\int_{1}^{x} \Delta(u)^{2} d u=c_{1} x^{3 / 2}-\frac{1}{4 \pi^{2}} x \log ^{2} x+c_{2} x \log x+O(x),
$$

where $c_{1}$ and $c_{2}$ are certain constants. In particular, it was suggested that the second and third terms on the right side of (1-2) come from the residues of $D_{2}(s)$ at $s=1$ [Furuya et al. 2010, Section 5].

In this paper, we first consider the Dirichlet series $Y(s)$ defined by

$$
Y(s)=\sum_{n=1}^{\infty} \frac{d(n) \Delta(n)}{n^{s}},
$$

which can be regarded as a modification of $D_{1}(s)$ and $D_{2}(s)$. We can easily see that the function $Y(s)$ is absolutely convergent in $\sigma>5 / 4$, similarly to $D_{1}(s)$, since $d(n)=O\left(n^{\varepsilon}\right)$ for an arbitrarily small positive number $\varepsilon$ and

$$
\sum_{n \leq x}|\Delta(n)|=O\left(x^{5 / 4}\right) .
$$

As for the other analytic properties of $Y(s)$, we obtain this:

Theorem 1. The Dirichlet series $Y(s)$ can be continued analytically to the region $\operatorname{Re} s>-1 / 3$ as a meromorphic function. In the region $\operatorname{Re} s \geq 1 / 2$, it has a simple pole at $s=1 / 2$ with

$$
\operatorname{Res}_{s=1 / 2} Y(s)=\frac{1}{16 \pi^{2}} \sum_{n=1}^{\infty} \frac{d(n)^{2}}{n^{3 / 2}},
$$

and it also has a pole of fourth order at $s=1$, whose Laurent expansion at $s=1$ is given by

$$
Y(s)=\frac{3}{\pi^{2}(s-1)^{4}}+\frac{12\left(\pi^{2} \gamma-3 \zeta^{\prime}(2)\right)}{\pi^{4}(s-1)^{3}}+\frac{a_{-2}}{(s-1)^{2}}+\frac{a_{-1}}{s-1}+\cdots,
$$


with some constants $a_{j}$, where $\zeta(s)$ denotes the Riemann zeta function. In the region $-1 / 3<\operatorname{Re} s<1 / 2$, the function $Y(s)$ has poles at $s=\rho / 2$ if $\rho$ satisfies the conditions $\zeta(\rho)=0$ and $\zeta(\rho / 2) \neq 0 .{ }^{*}$

We shall give the proof of this theorem in two ways; see Sections 3 and 7.

As the first application of Theorem 1, we shall study the Dirichlet series related to the coefficient of $\tilde{\Delta}(n)$ defined by

$$
\tilde{\Delta}(x)=\sum_{n \leq x}^{\prime} d(n)-x(\log x+2 \gamma-1)-\frac{1}{4},
$$

where $\sum_{n \leq x}^{\prime}$ indicates that the last term is to be halved if $x$ is an integer. Commonly this definition is used as the error term instead of (1-1). Many properties of $\Delta(x)$ also hold in the case $\tilde{\Delta}(x)$; for example, these functions have same upper and lower bounds as $x \rightarrow \infty$. For the mean value theorem, we can see that

$$
\int_{1}^{x} \Delta(u) d u=\frac{1}{4} x+O\left(x^{3 / 4}\right) \quad \text { and } \quad \int_{1}^{x} \tilde{\Delta}(u) d u=O\left(x^{3 / 4}\right)
$$

for $x \geq 1$, though the asymptotic behaviors, in particular the main terms of the higher power cases from 2 to 9, are the same. However, the difference between $\Delta(n)$ and $\tilde{\Delta}(n)$ for natural numbers $n$ is essential in the study of the "discrete" mean values. Actually, these functions are connected by the relation

$$
\tilde{\Delta}(n)=\Delta(n)-\frac{1}{2} d(n)-\frac{1}{4},
$$

for a natural number $n$; hence we have

$$
\sum_{n \leq x} \tilde{\Delta}(n)^{k}=\sum_{n \leq x} \Delta(n)^{k}+\sum_{b=0}^{k-1} \sum_{a=0}^{k-b} \frac{k !(-1)^{b-k} 2^{a+2 b-2 k}}{a ! b !(k-a-b) !} \sum_{n \leq x} d(n)^{a} \Delta(n)^{b},
$$

with a fixed natural number $k$ [Furuya 2007, Formula 5.1]. In view of this formula, studying the discrete mean values of $\tilde{\Delta}(n)$ will require that we understand the function $\sum_{n \leq x} d(n)^{a} \Delta(n)^{b}$. As noted in [Furuya 2007], it is very difficult to study this kind of sum in the case $a \geq 2$.

Now we consider the Dirichlet series

$$
\widetilde{D}_{j}(s)=\sum_{n=1}^{\infty} \frac{\tilde{\Delta}(n)^{j}}{n^{s}} \quad \text { for } j=1 \text { and } 2 .
$$

It is easily seen that these functions are absolutely convergent for $\sigma>5 / 4$ for $j=1$ and $\sigma>3 / 2$ for $j=2$, similarly to the cases of $D_{j}(s)$. For the other properties, we have the following corollary.

${ }^{*}$ Needless to say, the last condition $\zeta(\rho / 2) \neq 0$ holds if the Riemann Hypothesis is true. 
Corollary 1. (1) The function $\widetilde{D}_{1}(s)$ can be continued to the whole complex plane as a meromorphic function with a simple pole at $s=-2 n$ for a nonnegative integer $n$; in particular, this function is holomorphic at $s=1$. The residue of $\widetilde{D}_{1}(s)$ at $s=-2 n$ is the same as that of $D_{1}(s)$ and is given by

$$
\operatorname{Res}_{s=-2 n} \widetilde{D}_{1}(s)=-\frac{\zeta(-2 n-1)}{2 n+1} .
$$

(2) The function $\widetilde{D}_{2}(s)$ can be continued analytically as a meromorphic function to the region $\operatorname{Re} s>2 / 3$, where it has a simple pole at $s=3 / 2$ and a pole of fourth order at $s=1$. The residue of $\widetilde{D}_{2}(s)$ at $s=3 / 2$ is given by

$$
\operatorname{Res}_{s=3 / 2} \widetilde{D}_{2}(s)=\frac{1}{4 \pi^{2}} \sum_{n=1}^{\infty} \frac{d(n)^{2}}{n^{3 / 2}} .
$$

The proof of this corollary is based on the relation (1-3), Theorem 1, and the known results concerning $D_{j}(s)$ and $\zeta(s)$. Actually, we have by (1-3) that

$$
\begin{aligned}
& \widetilde{D}_{1}(s)=D_{1}(s)-\frac{1}{2} \zeta^{2}(s)-\frac{1}{4} \zeta(s), \\
& \widetilde{D}_{2}(s)=D_{2}(s)-\frac{1}{2} D_{1}(s)+\frac{1}{4} \zeta^{2}(s)+\frac{1}{16} \zeta(s)+\frac{\zeta^{4}(s)}{4 \zeta(2 s)}-Y(s) .
\end{aligned}
$$

The corollary follows immediately from these. (In fact, we need not use Theorem 1 to prove (1); we need only apply [Furuya et al. 2010, Theorem 1].)

Comparing this corollary with [Furuya et al. 2010, Theorems 1 and 2], we can see that the behaviors of $\widetilde{D}_{j}(s)$ and $D_{j}(s)$ are different. We also note that the residue of $\widetilde{D}_{2}(s)$ at $s=3 / 2$ is the same as that of $I_{2}(s)$, which is defined in the beginning of Section 2; see also Lemma 2 below.

We further study the properties of Dirichlet series related to $\Delta(x)$, especially the orders of $D_{2}(s)$ and $Y(s)$, whose analytic properties are poorly understood. Namely, their functional equations, approximate functional equations, and mean values are not known. It seems difficult to study the orders of these Dirichlet series in a satisfactory way. However:

Theorem 2. Let $s=\sigma+i$ be a complex variable. For $|t| \geq 2$, we have

$$
Y(s) \ll \begin{cases}1 & \text { for } \sigma>5 / 4, \\ |t|^{(5-4 \sigma) / 3} \log ^{5 / 2}|t| & \text { for } 1 / 2 \leq \sigma \leq 5 / 4 .\end{cases}
$$

Theorem 3. Let $s=\sigma+i$ t be a complex variable. For $|t| \geq 2$, we have

$$
D_{2}(s) \ll \begin{cases}1 & \text { for } \sigma>3 / 2, \\ \log |t| & \text { for } \sigma=3 / 2, \\ |t|^{3-2 \sigma} \log ^{4}|t| & \text { for } 1<\sigma<3 / 2 .\end{cases}
$$


These are obtained by using mean value theorems of $\Delta(x)$ and the PhragménLindelöf convexity theorem. The factor $\log ^{4}|t|$ in Theorem 3 corresponds to the error estimate of the mean square of $\Delta(x)$ [Preissmann 1988]. We can improve it slightly by using the recent result of Lau and Tsang [2009, Theorem 2], but for simplicity we use the result of Preissmann here.

Finally, as an application of Theorem 1, we will study an analytic continuation of a certain kind of multiple zeta function. Such functions are of current interest, especially those of the Euler-Zagier type

$$
\sum_{n_{1}<n_{2}<\cdots<n_{k}} \frac{1}{n_{1}^{s_{1}} n_{2}^{S_{2}} \ldots n_{k}^{s_{k}}} .
$$

As a generalization, one can consider two types of multiple series,

$$
\sum_{n_{1}<n_{2}<\cdots<n_{k}} \frac{a_{1}\left(n_{1}\right) a_{2}\left(n_{2}\right) \cdots a_{k}\left(n_{k}\right)}{n_{1}^{S_{1}} n_{2}^{S_{2}} \cdots n_{k}^{s_{k}}}
$$

and

$$
\sum_{n_{1}<n_{2}<\cdots<n_{k}} \frac{a_{1}\left(n_{1}\right) a_{2}\left(n_{2}-n_{1}\right) \cdots a_{k}\left(n_{k}-n_{k-1}\right)}{n_{1}^{s_{1}} n_{2}^{s_{2}} \cdots n_{k}^{s_{k}}},
$$

where $a_{j}(n)$ are certain arithmetical functions. Under suitable assumptions on the Dirichlet series $\sum_{n=1}^{\infty} a_{j}(n) n^{-s_{j}}$, the analytic properties for the multiple series of type (1-5) can be easily derived. Compared with (1-5), the series of type (1-4) is rather difficult, and it seems that [Akiyama and Ishikawa 2002] is the only character $\bmod q_{j}$ is treated in the case $a_{j}(n)=\chi_{j}(n)$; that paper made use of the periodicity of $\chi_{j}$ to reduce the problem to the multiple Hurwitz zeta function

$$
\sum_{n_{1}<n_{2}<\cdots<n_{k}} \frac{1}{\left(n_{1}+\alpha_{1}\right)^{s_{1}}\left(n_{2}+\alpha_{2}\right)^{s_{2}} \ldots\left(n_{k}+\alpha_{k}\right)^{s_{k}}} .
$$

The multiple series that we consider here is of the form

$$
D\left(s_{1}, s_{2}\right)=\sum_{m<n} \frac{d(m) d(n)}{m^{s_{1}} n^{s_{2}}} .
$$

For $\operatorname{Re} s_{2}>1$ and $\operatorname{Re}\left(s_{1}+s_{2}\right)>2$, the series in (1-6) is absolutely convergent and represents a holomorphic function in $s_{1}$ and $s_{2}$. Since the divisor function $d(n)$ is not periodic, we should adopt a different approach than Akiyama and Ishikawa.

In the case $s_{1}=s_{2}=s$, it is easy to see that $D(s, s)$ has an analytic continuation to the whole plane $\mathbb{C}$, since trivially

$$
D(s, s)=\frac{1}{2} \zeta(s)^{4}-\frac{\zeta(2 s)^{4}}{2 \zeta(4 s)}
$$


For general $s_{j}$, the analytic continuation of (1-6) is as follows:

Theorem 4. The multiple zeta function $D\left(s_{1}, s_{2}\right)$ can be continued analytically to a function meromorphic in the region in $\mathbb{C}^{2}$ given by

$$
\operatorname{Re} s_{1}+\operatorname{Re} s_{2}>\frac{1}{2} \text {. }
$$

To prove Theorem 4, we employ previous results about the Dirichlet series $D_{j}(s), Y(s)$, and $I_{j}(s)$ and their derivatives. More precisely, we will express $D\left(s_{1}, s_{2}\right)$ in terms of these functions and then use their analytic continuations. We can determine the singularities of $D\left(s_{1}, s_{2}\right)$ in the region $\operatorname{Re} s_{1}+\operatorname{Re} s_{2}>1 / 2$ by using the explicit formula (6-3) for $D\left(s_{1}, s_{2}\right)$. However, we shall omit the details of these properties since we would like to state the properties of $D\left(s_{1}, s_{2}\right)$ as simply as possible.

\section{Preliminaries}

Here we prepare some lemmas. The first concerns the analytic properties of the integrals

$$
I_{j}(s)=\int_{1}^{\infty} u^{-s} \Delta(u)^{j} d u \text { for } j=1 \text { and } 2 .
$$

We easily see that these integrals are absolutely convergent in the region $\sigma>5 / 4$ for $j=1$ and $\sigma>3 / 2$ for $j=2$.

Lemma 1 [Sitaramachandra Rao 1987]. The function $I_{1}(s)$ can be continued to the whole complex plane as a function holomorphic except for a simple pole at $s=1,{ }^{\dagger}$ and is expressed explicitly by

$$
I_{1}(s)=\frac{\zeta^{2}(s-1)}{s-1}-\frac{2 \gamma-1}{s-2}-\frac{1}{(s-2)^{2}} .
$$

Lemma 2 [Furuya et al. 2010, Lemma 4]. The function $I_{2}(s)$ can be continued analytically to the right half-plane $\sigma>2 / 3$. It has a simple pole at $s=3 / 2$ with residues

$$
\operatorname{Res}_{s=3 / 2} I_{2}(s)=\frac{1}{4 \pi^{2}} \sum_{n=1}^{\infty} \frac{d(n)^{2}}{n^{3 / 2}},
$$

while it has a triple pole at $s=1$.

We will need several results about sums of $\Delta(n)$.

Lemma 3. Let $\Delta(x)$ be the error term defined by (1-1). Then

$$
\sum_{n \leq x} \Delta(n)^{2}=c_{1} x^{3 / 2}+F(x)
$$

${ }^{\dagger}$ The function $I_{1}(s)$ is holomorphic at $s=2$, since the integral of $I_{1}(s)$ converges absolutely for $s=2$. (This can also be checked using the Laurent expansion around $s=2$ of the right side of (2-1).) 
with $F(x)=O\left(x \log ^{4} x\right)$, where $c_{1}$ is the constant defined in (1-2).

Proof. This formula can be proved directly by using [Furuya 2005, Theorem 1] and the asymptotic formula

$$
\int_{1}^{x} \Delta(u)^{2} d u=c_{1} x^{3 / 2}+O\left(x \log ^{4} x\right)
$$

due to Preissmann [1988].

\section{Lemma 4.}

$\sum_{n \leq x} d(n) \Delta(n)=\frac{1}{2} \sum_{n \leq x} d(n)^{2}+\frac{1}{2} \Delta(x)^{2}-\frac{1}{2}(2 \gamma-1)^{2}+\int_{1}^{x}(\log u+2 \gamma) \Delta(u) d u$.

We can write this sum explicitly as an asymptotic formula

$$
\sum_{n \leq x} d(n) \Delta(n)=\frac{1}{2 \pi^{2}} x \log ^{3} x+c_{3} x \log ^{2} x+c_{4} x \log x+c_{5} x+O\left(x^{3 / 4} \log x\right),
$$

with suitable constants $c_{3}, c_{4}$ and $c_{5}$.

Proof. The first formula is derived from [Furuya 2007, Theorem 1] by putting $f(n)=d(n)$, which implies $g(x)=x(\log x+2 \gamma-1)$ and $E(x)=\Delta(x)$. The second formula is [Furuya 2007, Corollary 1].

\section{The function $Y(s)$}

Let $N$ be a sufficiently large positive number and let

$$
Y_{N}(s)=\sum_{n \leq N} d(n) \Delta(n) n^{-s}
$$

for $\sigma>5 / 4$. Also put $g(x)=x(\log x+2 \gamma-1)$. Then by partial summation and the first formula of Lemma 4 , we have

$$
\begin{aligned}
& Y_{N}(s)= N^{-s} \sum_{n \leq N} d(n) \Delta(n)+s \int_{1}^{N} u^{-s-1} \sum_{n \leq u} d(n) \Delta(n) d u \\
&=s \int_{1}^{N} u^{-s-1}\left(\frac{1}{2} \sum_{n \leq u} d(n)^{2}+\frac{1}{2} \Delta(u)^{2}-\frac{1}{2}(2 \gamma-1)^{2}+\int_{1}^{u} g^{\prime}(v) \Delta(v) d v\right) d u \\
&+O\left(N^{1-\sigma} \log ^{3} N\right) .
\end{aligned}
$$

For the double integral on the right side, we have

$$
\begin{aligned}
\int_{1}^{N} u^{-s-1} \int_{1}^{u} g^{\prime}(v) \Delta(v) d v d u & =\int_{1}^{N} g^{\prime}(v) \Delta(v) \int_{v}^{N} u^{-s-1} d u d v \\
& =\frac{1}{s} \int_{1}^{N} u^{-s} g^{\prime}(u) \Delta(u) d u+O\left(N^{1-\sigma} \log N\right) .
\end{aligned}
$$


Furthermore, we have

$$
\sum_{n \leq x} d(n)^{2} n^{-s}=x^{-s} \sum_{n \leq x} d(n)^{2}+s \int_{1}^{x} u^{-s-1} \sum_{n \leq u} d(n)^{2} d u
$$

by partial summation; hence,

$$
s \int_{1}^{N} u^{-s-1} \sum_{n \leq u} d(n)^{2} d u=\sum_{n \leq N} d(n)^{2} n^{-s}+O\left(N^{1-\sigma} \log ^{3} N\right) .
$$

Therefore

$$
\begin{aligned}
Y_{N}(s)=\frac{1}{2} \sum_{n \leq N} d(n)^{2} n^{-s}+\frac{1}{2} s \int_{1}^{N} u^{-s-1} \Delta(u)^{2} d u-\frac{(2 \gamma-1)^{2}}{2} & \\
& +\int_{1}^{N} u^{-s} g^{\prime}(u) \Delta(u) d u+O\left(N^{1-\sigma} \log ^{3} N\right) .
\end{aligned}
$$

In the above formula, we let $N \rightarrow \infty$ and get

$$
Y(s)=\frac{\zeta(s)^{4}}{2 \zeta(2 s)}+\frac{s I_{2}(s+1)}{2}-\frac{(2 \gamma-1)^{2}}{2}+2 \gamma I_{1}(s)-I_{1}^{\prime}(s) .
$$

This expression holds for $\sigma>5 / 4$. But we can easily see, by (3-2) and the analytic properties of $\zeta(s), I_{j}(s)$ (for $\left.j=1,2\right)$ and $I_{1}^{\prime}(s)$, that $Y(s)$ is continued analytically from $\sigma>5 / 4$ to the region $\sigma>-1 / 3$.

Furthermore, we see that $Y(s)$ has poles at $s=1 / 2$ and $s=1$ in the region $\sigma \geq 1 / 2$. For $-1 / 3<\sigma<1 / 2$, the assertion in the theorem is easily derived from the right side of (3-2). The residue at $s=1 / 2$ is derived easily from Lemma 2, and the Laurent expansion of $Y(s)$ at $s=1$ is derived also by the right side of (3-2). This completes the proof of Theorem 1.

\section{The order of $Y(s)$}

In this section, we prove Theorem 2. Specifically, we determine the order of $Y(s)$ on the vertical lines $\sigma=1 / 2$ and $\sigma=5 / 4$ and apply the Phragmén-Lindelöf convexity theorem for $1 / 2 \leq \sigma \leq 5 / 4$.

First we consider the order of $Y(s)$ on the line $\sigma=1 / 2$. From (3-2), we have $Y\left(\frac{1}{2}+i t\right)=\frac{\zeta\left(\frac{1}{2}+i t\right)^{4}}{2 \zeta(1+2 i t)}+\frac{\frac{1}{2}+i t}{2} I_{2}\left(\frac{3}{2}+i t\right)-\frac{(2 \gamma-1)^{2}}{2}+2 \gamma I_{1}\left(\frac{1}{2}+i t\right)-I_{1}^{\prime}\left(\frac{1}{2}+i t\right)$.

It is easily seen that

$$
\frac{\zeta\left(\frac{1}{2}+i t\right)^{4}}{\zeta(1+2 i t)} \ll|t|^{2 / 3} \log ^{7}|t| \ll|t| .
$$


We also have $I_{1}\left(\frac{1}{2}+i t\right) \ll|t|^{-1}\left|\zeta\left(-\frac{1}{2}+i t\right)\right|^{2} \ll|t|$ from Lemma 1 , and

$$
I_{1}^{\prime}\left(\frac{1}{2}+i t\right) \ll|t|^{-1}\left|\zeta\left(-\frac{1}{2}+i t\right) \zeta^{\prime}\left(-\frac{1}{2}+i t\right)\right| \ll|t| \log |t|
$$

similarly. So it remains to consider $I_{2}\left(\frac{3}{2}+i t\right)$ :

Lemma 5. $I_{2}\left(\frac{3}{2}+i t\right) \ll \log |t|$ as $|t| \rightarrow \infty$.

Proof. Assume $\sigma>3 / 2$, and let $X$ be a large parameter. Splitting the integral at $X$, we have

$$
I_{2}(s)=\int_{1}^{X} u^{-s} \Delta(u)^{2} d u+\int_{X}^{\infty} u^{-s} \Delta(u)^{2} d u=: J_{X}^{(1)}(s)+J_{X}^{(2)}(s) .
$$

Using the mean value estimate (2-2) and integration by parts, we have

$$
\begin{aligned}
J_{X}^{(2)}(s) & =\left[u^{-s}\left(c_{1} u^{3 / 2}+O\left(u \log ^{4} u\right)\right)\right]_{X}^{\infty}+s \int_{X}^{\infty} u^{-s-1}\left(c_{1} u^{3 / 2}+O\left(u \log ^{4} u\right)\right) d u \\
& =\frac{3 c_{1}}{2 s-3} X^{-s+3 / 2}+O\left(X^{1-\sigma} \log ^{4} X\right)+O\left(|t| \int_{X}^{\infty} u^{-\sigma} \log ^{4} u d u\right) .
\end{aligned}
$$

The integral in the last term converges absolutely in the region $\sigma>1$ and is estimated as $O\left(|t| X^{1-\sigma} \log ^{4} X\right)$. Hence we have

$$
J_{X}^{(2)}\left(\frac{3}{2}+i t\right) \ll|t|^{-1}+X^{-1 / 2} \log ^{4} X+|t| X^{-1 / 2} \log ^{4} X .
$$

Meanwhile,

$$
J_{X}^{(1)}\left(\frac{3}{2}+i t\right) \ll \int_{1}^{X} u^{-3 / 2} \Delta^{2}(u) d u \ll \log X .
$$

By taking, for example, $X=|t|^{3}$, we obtain the lemma.

From these estimates, we obtain

$$
Y\left(\frac{1}{2}+i t\right) \ll|t| \log |t| .
$$

Next we consider the order on the line $\sigma=5 / 4$. Assuming first that $\sigma>5 / 4$ as usual, we define

$$
E_{N}(s)=Y(s)-Y_{N}(s)=\sum_{n>N} \frac{d(n) \Delta(n)}{n^{s}},
$$

where $Y_{N}(s)$ is the function defined by (3-1).

Using partial summation and the second formula in Lemma 4, we have

$$
\begin{aligned}
E_{N}(s)=- & N^{-s}\left(\frac{1}{2 \pi^{2}} N \log ^{3} N+c_{3} N \log ^{2} N+c_{4} N \log N+c_{5} N\right) \\
& +s \int_{N}^{\infty} u^{-s-1}\left(\frac{1}{2 \pi^{2}} u \log ^{3} u+c_{3} u \log ^{2} u+c_{4} u \log u+c_{5} u\right) d u \\
& +O\left(|t| N^{3 / 4-\sigma} \log N\right)
\end{aligned}
$$




$$
\begin{aligned}
=\frac{1}{2 \pi^{2}(s-1)} N^{1-s} \log ^{3} N+\left(\frac{3 s}{2 \pi^{2}(s-1)^{2}}+\frac{c_{3}}{s-1}\right) N^{1-s} \log ^{2} N \\
+\left(\frac{6 s}{2 \pi^{2}(s-2)^{3}}+\frac{2 c_{3} s}{(s-1)^{2}}+\frac{c_{4}}{s-1}\right) N^{1-s} \log N \\
+\left(\frac{6 s}{2 \pi^{2}(s-1)^{4}}+\frac{2 c_{3} s}{(s-1)^{3}}+\frac{c_{4} s}{(s-1)^{2}}+\frac{c_{5}}{s-1}\right) N^{1-s} \\
+O\left(|t| N^{3 / 4-\sigma} \log N\right) .
\end{aligned}
$$

Hence, we get the estimate

$$
E_{N}(s) \ll \frac{N^{1-\sigma} \log ^{3} N}{|t|}+|t| N^{3 / 4-\sigma} \log N
$$

for $\sigma>5 / 4$. Note that (4-2) holds true for $\sigma>3 / 4$.

On the other hand, the first part of this division can be estimated as

$$
Y_{N}\left(\frac{5}{4}+i t\right) \ll\left(\sum_{n \leq N} \frac{d(n)^{2}}{n}\right)^{1 / 2}\left(\sum_{n \leq N} \frac{\Delta(n)^{2}}{n^{3 / 2}}\right)^{1 / 2} \ll \log ^{5 / 2} N .
$$

Taking $N=|t|^{2}$, we then get

$$
Y\left(\frac{5}{4}+i t\right) \ll \log ^{5 / 2}|t| .
$$

By (4-1), (4-3) and the Phragmén-Lindelöf principle, we obtain

$$
Y(\sigma+i t) \ll|t|^{(5-4 \sigma) / 3} \log ^{5 / 2}|t| \text { for } 1 / 2 \leq \sigma \leq 5 / 4,
$$

which completes the proof of Theorem 3.

\section{The order of $D_{2}(s)$}

Let $\sigma>3 / 2$. We divide the infinite series as

$$
\sum_{n=1}^{\infty} \frac{\Delta(n)^{2}}{n^{s}}=\left(\sum_{n \leq N}+\sum_{n>N}\right) \frac{\Delta(n)^{2}}{n^{s}}=D_{2, N}^{(1)}(s)+D_{2, N}^{(2)}(s) .
$$

By using partial summation and Lemma 2, we have

$$
\begin{aligned}
D_{2, N}^{(2)}(s) & =\frac{\frac{3}{2} c_{1}}{s-\frac{3}{2}} N^{-s+3 / 2}-N^{-s} F(N)+s \int_{N}^{\infty} u^{-s-1} F(u) d u \\
& =\frac{\frac{3}{2} c_{1}}{s-\frac{3}{2}} N^{-s+3 / 2}+O\left(|s| N^{1-\sigma} \log ^{4} N\right) .
\end{aligned}
$$

This estimate actually holds for $\sigma>1$, and thus this formula gives the analytic continuation of $D_{2, N}^{(2)}(s)$ from $\sigma>3 / 2$ into $\sigma>1$. 
We treat the case $s=\frac{3}{2}+i t$. By (5-1), we have

$$
D_{2, N}^{(2)}\left(\frac{3}{2}+i t\right) \ll|t|^{-1}+|t| N^{-1 / 2} \log ^{4} N .
$$

We have, by partial summation and Lemma 2 again,

$$
D_{2, N}^{(1)}\left(\frac{3}{2}+i t\right) \ll \sum_{n \leq N} \frac{\Delta(n)^{2}}{n^{3 / 2}} \ll \log N .
$$

Hence, by taking $N=|t|^{3}$, we have $D_{2}\left(\frac{3}{2}+i t\right) \ll \log |t|$. This estimate gives the second assertion of Theorem 3.

To prove the third, we first consider the case $s=1+\varepsilon+i t$, where $\varepsilon$ is a fixed positive small number. By (5-1), we get

$$
D_{2, N}^{(2)}(1+\varepsilon+i t) \ll|t|^{-1} N^{1 / 2-\varepsilon}+|t| N^{-\varepsilon} \log ^{4} N,
$$

and

$$
D_{2, N}^{(1)}(1+\varepsilon+i t) \ll N^{1 / 2-\varepsilon} .
$$

Hence, by taking $N=|t|^{2}$, we get $D_{2}(1+\varepsilon+i t) \ll|t|^{1-2 \varepsilon} \log ^{4}|t|$.

Applying the Phragmén-Lindelöf convexity principle, we obtain

$$
D_{2}(\sigma+i t) \ll|t|^{3-2 \sigma} \log ^{4}|t|
$$

for $1<\sigma<3 / 2$. This completes the proof of Theorem 3 .

\section{Proof of Theorem 4}

Let

$$
S\left(s_{1}, s_{2}\right)=\sum_{m \leq n} \frac{d(m) d(n)}{m^{s_{1}} n^{s_{2}}} .
$$

To prove Theorem 4, it is enough to consider the series $S\left(s_{1}, s_{2}\right)$, since

$$
D\left(s_{1}, s_{2}\right)=S\left(s_{1}, s_{2}\right)-\frac{\zeta^{4}\left(s_{1}+s_{2}\right)}{\zeta\left(2\left(s_{1}+s_{2}\right)\right)} .
$$

Let $s_{j}=\sigma_{j}+i t_{j}$ be complex variables. First assume that $\sigma_{1}>1$ and $\sigma_{2}>1$. For a large positive number $N$, we consider the finite sum

$$
S_{N}\left(s_{1}, s_{2}\right):=\sum_{m \leq n \leq N} \frac{d(m) d(n)}{m^{s_{1}} n^{s_{2}}} .
$$

By partial summation and (1-1), we have

$$
S_{N}\left(s_{1}, s_{2}\right)=\sum_{n \leq N} \frac{d(n)}{n^{s_{2}}} \sum_{m \leq n} \frac{d(m)}{m^{s_{1}}}
$$




$$
\begin{array}{r}
=\sum_{n \leq N} \frac{d(n)}{n^{s_{2}}}\left(\frac{1}{n^{s_{1}}} \sum_{m \leq n} d(m)+s_{1} \int_{1}^{n} u^{-s_{1}-1}\left(\sum_{m \leq u} d(m)\right) d u\right) \\
=\sum_{n \leq N} \frac{d(n)(g(n)+\Delta(n))}{n^{s_{1}+s_{2}}}+s_{1} \sum_{n \leq N} \frac{d(n)}{n^{s_{2}}} \int_{1}^{n} u^{-s_{1}-1} g(u) d u \\
+s_{1} \sum_{n \leq N} \frac{d(n)}{n^{s_{2}}} \int_{1}^{n} u^{-s_{1}-1} \Delta(u) d u,
\end{array}
$$

where $g(u)=u(\log u+2 \gamma-1)$ as before. In the last term above, split the integral as $\int_{1}^{n}=\int_{1}^{N}-\int_{n}^{N}$. Then interchange the order of integral and summation and use partial summation and (1-1) again. We get eight terms:

$$
\begin{aligned}
S_{N}\left(s_{1}, s_{2}\right)=\sum_{n \leq N} & \frac{d(n) g(n)}{n^{s_{1}+s_{2}}}+\sum_{n \leq N} \frac{d(n) \Delta(n)}{n^{s_{1}+s_{2}}}+s_{1} \sum_{n \leq N} \frac{d(n)}{n^{s_{2}}} \int_{1}^{n} u^{-s_{1}-1} g(u) d u \\
& +s_{1}\left(\sum_{n \leq N} \frac{d(n)}{n^{s_{2}}}\right) \int_{1}^{N} u^{-s_{1}-1} \Delta(u) d u \\
& -s_{1} \int_{1}^{N} u^{-s_{1}-s_{2}-1} \Delta(u) g(u) d u-s_{1} \int_{1}^{N} u^{-s_{1}-s_{2}-1} \Delta(u)^{2} d u \\
& -s_{1} s_{2} \int_{1}^{N} u^{-s_{1}-1} \Delta(u) \int_{1}^{u} v^{-s_{2}-1} g(v) d v d u \\
& -s_{1} s_{2} \int_{1}^{N} u^{-s_{1}-1} \Delta(u) \int_{1}^{u} v^{-s_{2}-1} \Delta(v) d v d u,
\end{aligned}
$$

which we define as $\sum_{j=1}^{8} I_{j, N}\left(s_{1}, s_{2}\right)$. We consider each $I_{j}\left(s_{1}, s_{2}\right)$ as $N \rightarrow \infty$. It is easy to see that

$$
\lim _{N \rightarrow \infty} I_{1, N}\left(s_{1}, s_{2}\right)=-\left(\zeta^{2}\right)^{\prime}\left(s_{1}+s_{2}-1\right)+(2 \gamma-1) \zeta^{2}\left(s_{1}+s_{2}-1\right) .
$$

By elementary calculations, we have

$$
\begin{aligned}
\lim _{N \rightarrow \infty} I_{3, N}\left(s_{1}, s_{2}\right)=\frac{s_{1}}{s_{1}-1}\left(\zeta^{2}\right)^{\prime}\left(s_{1}+s_{2}-1\right) & \\
& \quad-s_{1}\left(\frac{1}{\left(s_{1}-1\right)^{2}}+\frac{2 \gamma-1}{s_{1}-1}\right)\left(\zeta^{2}\left(s_{1}+s_{2}-1\right)-\zeta^{2}\left(s_{2}\right)\right) .
\end{aligned}
$$

The terms $I_{j, N}\left(s_{1}, s_{2}\right)$ for $j=2,4,5,6,7$ can be written in terms of the functions $Y(s), I_{1}(s), I_{1}^{\prime}(s)$ and $I_{2}(s)$. In fact, we have

$$
\begin{aligned}
& \lim _{N \rightarrow \infty} I_{2, N}\left(s_{1}, s_{2}\right)=Y\left(s_{1}+s_{2}\right), \\
& \lim _{N \rightarrow \infty} I_{4, N}\left(s_{1}, s_{2}\right)=s_{1} \zeta^{2}\left(s_{2}\right) I_{1}\left(s_{1}+1\right),
\end{aligned}
$$




$$
\begin{aligned}
& \lim _{N \rightarrow \infty} I_{5, N}\left(s_{1}, s_{2}\right)=-s_{1}\left(-I_{1}^{\prime}\left(s_{1}+s_{2}\right)+(2 \gamma-1) I_{1}\left(s_{1}+s_{2}\right)\right), \\
& \lim _{N \rightarrow \infty} I_{6, N}\left(s_{1}, s_{2}\right)=-s_{1} I_{2}\left(s_{1}+s_{2}+1\right),
\end{aligned}
$$

and

$$
\begin{aligned}
\lim _{N \rightarrow \infty} I_{7, N}\left(s_{1}, s_{2}\right)=-\frac{s_{1} s_{2}}{s_{2}-1} & I_{1}^{\prime}\left(s_{1}+s_{2}\right) \\
& \quad+s_{1} s_{2}\left(\frac{1}{\left(s_{2}-1\right)^{2}}+\frac{2 \gamma-1}{s_{2}-1}\right)\left(I_{1}\left(s_{1}+s_{2}\right)-I_{1}\left(s_{1}+1\right)\right) .
\end{aligned}
$$

By Theorem 1 and Lemmas 1 and 2, the terms $\lim _{N \rightarrow \infty} I_{j, N}\left(s_{1}, s_{2}\right)$ for $j=1, \ldots, 7$ can be continued meromorphically to the region $\sigma_{1}+\sigma_{2}>-1 / 3$.

We treat the term $I_{8, N}\left(s_{1}, s_{2}\right)$ with the following lemma.

Lemma 6. Let

$$
I^{(N)}\left(s_{1}, s_{2}\right)=\int_{1}^{N} u^{-s_{1}-1} \Delta(u) \int_{u}^{N} v^{-s_{2}-1} \Delta(v) d v d u,
$$

and

$$
I\left(s_{1}, s_{2}\right)=\lim _{N \rightarrow \infty} I^{(N)}\left(s_{1}, s_{2}\right) .
$$

Then I $\left(s_{1}, s_{2}\right)$ defines a holomorphic function in the region $\sigma_{2}>\frac{1}{4}$ and $\sigma_{1}+\sigma_{2}>\frac{1}{2}$. Proof. In the region $\sigma_{2}>\frac{1}{4}$ and $\sigma_{1}+\sigma_{2}>\frac{1}{2}$,

$$
I^{(N)}\left(s_{1}, s_{2}\right) \ll \int_{1}^{N} u^{-\sigma_{1}-1}|\Delta(u)| u^{-\sigma_{2}+1 / 4} d u \ll 1,
$$

since

$$
\int_{a}^{b} u^{\beta}|\Delta(u)| d u \ll a^{\beta+5 / 4}
$$

for $a \leq b$ and $\beta<-5 / 4$. The lemma follows immediately.

Now we consider the double integral

$$
J_{N}\left(s_{1}, s_{2}\right)=\int_{1}^{N} u^{-s_{1}-1} \Delta(u) \int_{1}^{u} v^{-s_{2}-1} \Delta(v) d v d u .
$$

Splitting the innermost integral in $J_{N}\left(s_{1}, s_{2}\right)$ as $\int_{1}^{u}=\int_{1}^{N}-\int_{u}^{N}$, we have

$$
J_{N}\left(s_{1}, s_{2}\right)=\int_{1}^{N} u^{-s_{1}-1} \Delta(u) d u \int_{1}^{N} u^{-s_{2}-1} \Delta(u) d u-I^{(N)}\left(s_{1}, s_{2}\right) .
$$

In $\sigma_{2}>\frac{1}{4}$ and $\sigma_{1}+\sigma_{2}>\frac{1}{2}$, we have

$$
J\left(s_{1}, s_{2}\right):=\lim _{N \rightarrow \infty} J_{N}\left(s_{1}, s_{2}\right)=I_{1}\left(s_{1}+1\right) I_{1}\left(s_{2}+1\right)-I\left(s_{1}, s_{2}\right) .
$$

Hence, $J\left(s_{1}, s_{2}\right)$ is a meromorphic function there by Lemmas 1 and 6 . 
On the other hand, by the symmetric property

$$
J_{N}\left(s_{1}, s_{2}\right)+J_{N}\left(s_{2}, s_{1}\right)=\int_{1}^{N} u^{-s_{1}-1} \Delta(u) d u \int_{1}^{N} u^{-s_{2}-1} \Delta(u) d u,
$$

we obtain

$$
J\left(s_{1}, s_{2}\right)=I_{1}\left(s_{1}+1\right) I_{1}\left(s_{2}+1\right)-J\left(s_{2}, s_{1}\right) .
$$

By applying the above argument on $I\left(s_{2}, s_{1}\right)$, we see that $J\left(s_{1}, s_{2}\right)$ is also defined in the region $\sigma_{1}>\frac{1}{4}$ and $\sigma_{1}+\sigma_{2}>\frac{1}{2}$. Therefore we conclude that

$$
\lim _{N \rightarrow \infty} I_{8, N}\left(s_{1}, s_{2}\right)=-s_{1} s_{2} J\left(s_{1}, s_{2}\right)
$$

is meromorphic in $\sigma_{1}+\sigma_{2}>\frac{1}{2}$. This completes the proof of Theorem 4 .

More concretely, the explicit form of the analytic continuation of $S\left(s_{1}, s_{2}\right)$ is given by

$$
\begin{aligned}
& S\left(s_{1}, s_{2}\right)=Y\left(s_{1}+s_{2}\right)-s_{1} I_{2}\left(s_{1}+s_{2}+1\right)-s_{1} s_{2} I_{1}\left(s_{1}+1\right) I_{1}\left(s_{2}+1\right) \\
& -\frac{s_{1}}{s_{2}-1} I_{1}^{\prime}\left(s_{1}+s_{2}\right)+s_{1}\left(\frac{s_{2}}{\left(s_{2}-1\right)^{2}}+\frac{2 \gamma-1}{s_{2}-1}\right) I_{1}\left(s_{1}+s_{2}\right) \\
& +s_{1} \zeta^{2}\left(s_{2}\right) I_{1}\left(s_{1}+1\right)-s_{1} s_{2}\left(\frac{1}{\left(s_{2}-1\right)^{2}}+\frac{2 \gamma-1}{s_{2}-1}\right) I_{1}\left(s_{1}+1\right) \\
& +\frac{1}{s_{1}-1}\left(\zeta^{2}\right)^{\prime}\left(s_{1}+s_{2}-1\right)-\left(\frac{s_{1}}{\left(s_{1}-1\right)^{2}}+\frac{2 \gamma-1}{s_{1}-1}\right) \zeta^{2}\left(s_{1}+s_{2}-1\right) \\
& +s_{1}\left(\frac{1}{\left(s_{1}-1\right)^{2}}+\frac{2 \gamma-1}{s_{1}-1}\right) \zeta^{2}\left(s_{2}\right)+s_{1} s_{2} I\left(s_{1}, s_{2}\right) \text {. }
\end{aligned}
$$

From this formula, we can determine the locations of singularities of $S\left(s_{1}, s_{2}\right)$, and thus $D\left(s_{1}, s_{2}\right)$ by (6-1), but we omit the details of this topic here.

\section{An alternative approach to Theorem 1}

We now give a proof of Theorem 1 by approaching (3-2) differently. In fact, we will not use the first result in Lemma 4, which is an identity for $\sum_{n \leq x} d(n) \Delta(n)$.

Let $Y_{N}(s)$ and $g(x)$ be defined as above. By (1-1), we have

$$
\begin{aligned}
& Y_{N}(s)=\sum_{n \leq N} \frac{d(n)}{n^{s}}\left(\sum_{m \leq n} d(m)-g(n)\right) \\
& =\left(\sum_{n \leq N} d(n)\right)\left(\sum_{n \leq N} \frac{d(n)}{n^{s}}\right)-\sum_{m \leq N}\left(d(m) \sum_{n \leq m} \frac{d(n)}{n^{s}}\right)+\sum_{n \leq N} \frac{d(n)^{2}}{n^{s}}-\sum_{n \leq N} \frac{d(n)}{n^{s}} g(n) .
\end{aligned}
$$


Further, since

$$
\begin{aligned}
\sum_{m \leq N} d(m) \sum_{n \leq m} \frac{d(n)}{n^{s}}=\sum_{m \leq N} \frac{d(m)}{m^{s}}(g(m) & +\Delta(m)) \\
& +s \sum_{m \leq N} d(m) \int_{1}^{m} u^{-s-1}(g(u)+\Delta(u)) d u
\end{aligned}
$$

by partial summation, we have, for $\sigma>5 / 4$,

$$
\begin{aligned}
2 Y_{N}(s)-\frac{\zeta^{4}(s)}{\zeta(2 s)}= & \left(\sum_{n \leq N} d(n)\right)\left(\sum_{n \leq N} \frac{d(n)}{n^{s}}\right)-2 \sum_{n \leq N} \frac{d(n)}{n^{s}} g(n) \\
& -s \sum_{m \leq N} d(m) \int_{1}^{m} u^{-s-1}(g(u)+\Delta(u)) d u+O\left(N^{1-\sigma} \log ^{3} N\right) .
\end{aligned}
$$

We now consider the transformation of $\int_{1}^{N} u^{-s} \Delta^{2}(u) d u$. We have by (1-1)

$$
\begin{aligned}
\int_{1}^{N} u^{-s} \Delta^{2}(u) d u & =\int_{1}^{N} u^{-s} \Delta(u)\left(\sum_{n \leq u} d(n)-g(u)\right) d u \\
& =\sum_{n \leq N} d(n) \int_{n}^{N} u^{-s} \Delta(u) d u-\int_{1}^{N} u^{-s} \Delta(u) g(u) d u \\
& =\left(\int_{1}^{N} u^{-s} \Delta(u) d u\right) \sum_{n \leq N} d(n)-\sum_{n \leq N} d(n) \int_{1}^{n} u^{-s} \Delta(u) d u \\
& -\int_{1}^{N} u^{-s} \Delta(u) g(u) d u .
\end{aligned}
$$

We obtain by this formula, and by applying partial summation to $\sum_{n \leq N} d(n) n^{-s}$, $\sum_{n \leq N} d(n) g(n) n^{-s}$, and $\sum_{n \leq N} d(n) \int_{1}^{n} u^{-s-1} g(u) d u$, that

$$
\begin{aligned}
2 Y_{N}(s)- & \frac{\zeta^{4}(s)}{\zeta(2 s)}-s I_{2}(s+1)+2 I_{1}^{\prime}(s)-4 \gamma I_{1}(s) \\
= & \left(N^{-s} \sum_{n \leq N} d(n)-2 N^{-s} g(N)\right) \sum_{n \leq N} d(n)+s \int_{1}^{N} u^{-s-1} g(u)^{2} d u \\
& \quad+2 \int_{1}^{N} g(u)\left(u^{-s} g^{\prime}(u)-s u^{-s-1} g(u)\right) d u+O\left(N^{5 / 4-\sigma} \log N\right)
\end{aligned}
$$

for $\sigma>5 / 4$. Furthermore, by applying the estimate $\Delta(x)=O\left(x^{1 / 3}\right)$ and the formula

$$
2 \int_{1}^{N} u^{-s} g(u) g^{\prime}(u) d u=N^{-s} g(N)^{2}-(2 \gamma-1)^{2}+s \int_{1}^{N} u^{-s-1} g(u)^{2} d u,
$$


which has been proved by integration by parts, we obtain $2 Y_{N}(s)-\frac{\zeta^{4}(s)}{\zeta(2 s)}-s I_{2}(s+1)+2 I_{1}^{\prime}(s)-4 \gamma I_{1}(s)=-(2 \gamma-1)^{2}+O\left(N^{5 / 4-\sigma} \log N\right)$ for $\sigma>5 / 4$. Thus, as $N$ tends toward infinity, we obtain again (3-2).

\section{References}

[Akiyama and Ishikawa 2002] S. Akiyama and H. Ishikawa, "On analytic continuation of multiple $L$-functions and related zeta-functions", pp. 1-16 in Analytic number theory (Beijing/Kyoto, 1999), edited by C. Jia and K. Matsumoto, Dev. Math. 6, Kluwer, Dordrecht, 2002. MR 2003b:11093 Zbl 1028.11058

[Furuya 2005] J. Furuya, "On the average orders of the error term in the Dirichlet divisor problem", J. Number Theory 115:1 (2005), 1-26. MR 2006h:11117 Zbl 1089.11055

[Furuya 2007] J. Furuya, "On the summatory function of a product of an arithmetical function and its relevant error term”, Ann. Sci. Math. Québec 31:2 (2007), 165-185. MR 2009k:11148 Zbl 05529025

[Furuya et al. 2010] J. Furuya, Y. Tanigawa, and W. Zhai, "Dirichlet series obtained from the error term in the Dirichlet divisor problem", preprint, 2010. To appear in Monatshefte Math.

[Lau and Tsang 1995] Y.-K. Lau and K.-M. Tsang, "Mean square of the remainder term in the Dirichlet divisor problem", J. Théor. Nombres Bordeaux 7:1 (1995), 75-92. Les Dix-huitièmes Journées Arithmétiques (Bordeaux, 1993). MR 98k:11126 Zbl 0844.11059

[Lau and Tsang 2009] Y.-K. Lau and K.-M. Tsang, "On the mean square formula of the error term in the Dirichlet divisor problem", Math. Proc. Cambridge Philos. Soc. 146:2 (2009), 277-287. MR 2009k:11149 Zbl 05532374

[Preissmann 1988] E. Preissmann, "Sur la moyenne quadratique du terme de reste du problème du cercle”, C. R. Acad. Sci. Paris Sér. I Math. 306:4 (1988), 151-154. MR 89e:11056 Zbl 0654.10042

[Sitaramachandra Rao 1987] R. Sitaramachandra Rao, "An integral involving the remainder term in the Piltz divisor problem”, Acta Arith. 48:1 (1987), 89-92. MR 88h:11068

Received April 1, 2009. Revised September 14, 2009.

JUN FURUYA

DEPARTMENT OF INTEgRATEd ARTS AND SCIENCE

OKINAWA National College of TeCHNOLOGY

NAGO, OKINAWA, 905-2192

JAPAN

jfuruya@okinawa-ct.ac.jp

YoshIO TANIGAWA

Graduate SCHOOL OF MATHEMATICS

NAGOYA UNIVERSITY

NAGOYA, 464-8602

JAPAN

tanigawa@math.nagoya-u.ac.jp 